\title{
DEVELOPMENT OF HUMANOIDS AND NEW BUSINESS STRUCTURE
}

Hirochika Inoue

Digital Human Research Center, AIST, Japan

This talk introduces some of the recent research and development of humanoid, and discusses the problems, approaches and applications for the next generation humanoid.

First, the humanoid research efforts at the University of Tokyo group, which the author has long been committed, are introduced. It includes various small robots created by the remote-brained approach, full-size humanoids designed as integrated intelligent systems, and a humanoid with a flexible spine structure. Second, the results of a Japanese national project for humanoid robotics will be shown. This five-year project, referred to as the HRP project, was carried out as the platform-based studies for humanoid and human friendly robotic applications. We have succeeded in building a new humanoid platform to serve as technical infrastructure for R\&D. The project has also challenged to realize five application scenarios, such as humanoids performing plant inspection routines, humanoid service workers in hospital environments, humanoid drivers that can operate ordinary construction machinery, cooperative tasks with human workers in outdoor environments, and controlling a home-sitting humanoid operated remotely using a PDA.

The new hardware platform HRP-2 and the software platform OpenHRP provide a common research tool not only for fundamental scientific studies but also for practical application studies. The humanoid platform changes a starting point of our humanoid $\mathrm{R} \& \mathrm{D}$, enables new approaches, opens new 
possibilities of humanoid related business, and contributes to develop the human-robot symbiotic society.

Technical problems to be solved, approaches for further developments, sorts of applications, business models, and a roadmap, towards the next generation, will be discussed. And the structure of future robot business will be figured out. 